\title{
A Comparative Study of Fetomaternal Outcome of Elective Cesarean and Vaginal Birth after Cesarean Section in a Tertiary Care Center in South India
}

\author{
Sathya Prabha Jagannathan ${ }^{1}$, Hingmire M Sunil ${ }^{2}$, Prema Nalligounder ${ }^{3}$
}

\begin{abstract}
Aims and objectives: To provide evidence-based information to choose the mode of delivery following a single cesarean section in a tertiary center in South India. The objective is to study the success rate, safety, and efficacy of vaginal birth after cesarean section (VBAC) by comparing the maternal and perinatal outcome with the elective repeat cesarean section (ERCS).

Materials and methods: This was a prospective, cohort study done over a period of 12 months in a tertiary care center. Based on the patients' preference, a total of 211 women who satisfied the inclusion criteria were divided into two groups-either trial of labor after cesarean section (TOLAC) or ERCS group.

Results: Success rate of VBAC was found to be $47.9 \%$. About $15 \%$ of failed VBAC was due to the tendency to abandon TOLAC midway. Although the maternal complications were found to be higher in TOLAC, $p$ value (0.347) was not found to be significant. There was no increased risk of neonatal morbidity and mortality in the TOLAC group when compared to ERCS $(p=0.814)$. There was also no difference found in Apgar scores $(<7)$ at 5 minutes and newborn intensive care unit (NICU) admissions in the TOLAC group and in the ERCS group $(p=0.899)$.

Conclusion: The success rate of VBAC was found to be $47.9 \%$. There was no significant risk of maternal and perinatal complications compared to ERCS.

Clinical significance: Although VBAC was found to be successful in developed countries, we need more of studies in our Indian setup with the local population to improve the quality of health care and create awareness among patients. This present study might boost the obstetricians in the tertiary care setup to counsel more for TOLAC.

Keywords: Cohort study, Elective repeat cesarean section, Maternal and neonatal outcome, Trial of labor after cesarean section.

Journal of South Asian Federation of Obstetrics and Gynaecology (2021): 10.5005/jp-journals-10006-1958
\end{abstract}

\section{INTRODUCTION}

Mode of delivery following a previous cesarean section is of major concern today. Due to the rising cesarean section rate and its associated complications, we need to pause and think of methods to improve normal labor, especially in a low-resource country like India with many small health care providers who might not be able to strictly adhere to the guidelines. The cesarean rate has risen from 21\% in 1996 to 32.9\% in 2009.1,2 The 2010 National Institutes of Health Consensus Conference on VBAC highlighted high-grade evidence that maternal mortality risk is decreased by VBAC compared with a repeat cesarean (3.8 vs 13.4 of 100,000$){ }^{2}$ Data suggest decreasing the primary cesarean delivery rate and increasing the VBAC rate as key strategies to decrease the cesarean rate. ${ }^{3}$ In pregnancies complicated by a history of previous cesarean section, both trial of labor after cesarean section (TOLAC) and ERCS are safe options for delivery with overall low absolute rates of adverse maternal or fetal outcomes. Although a repeat cesarean section is associated with increased maternal risks, most of these risks can be anticipated. In comparison, the success of TOLAC is unpredictable. If a VBAC is achieved, it confers lowest risks to both the mother and the infant, but, if the TOLAC fails, there is a greater risk to both. ${ }^{4}$ So, to reduce the overall morbidity and to improve the success rate of TOLAC, patient selection should be done with utmost care. Potential risk and benefits of both TOLAC and elective repeat cesarean section (ERCS) should be discussed with the patient and documented. Discussion should consider individual characteristics that affect the likelihood of complications associated with TOLAC \begin{tabular}{l}
\hline \hline 1,3 Department of Obstetrics and Gynaecology, Karpagam Faculty of \\
Medical Science and Research, Coimbatore, Tamil Nadu, India \\
2Department of Obstetrics and Gynaecology, G Kuppuswamy Naidu \\
Memorial Hospital, Coimbatore, Tamil Nadu, India \\
Corresponding Author: Sathya Prabha Jagannathan, Department of \\
Obstetrics and Gynaecology, Karpagam Faculty of Medical Science \\
and Research, Coimbatore, Tamil Nadu, India, Phone: +91 9943803262, \\
e-mail: sathyapraba.mdu@gmail.com \\
How to cite this article: Jagannathan SP, Sunil HM, Nalligounder P. \\
A Comparative Study of Fetomaternal Outcome of Elective Cesarean \\
and Vaginal Birth after Cesarean Section in a Tertiary Care Center in \\
South India. J South Asian Feder Obst Gynae 2021;13(5):283-287. \\
Source of support: Nil \\
Conflict of interest: None
\end{tabular}

and ERCS, so that a woman can choose her intended route of delivery. ${ }^{5}$ VBAC offers the advantage of avoiding major abdominal surgery and has lower rates of hemorrhage, thromboembolism, infection, and shorter recovery period than women who have an ERCS. ${ }^{6}$ Additionally, for those considering future pregnancies, VBAC may decrease the risk of maternal consequences related to multiple cesarean deliveries (e.g., hysterectomy, bowel or bladder injury, transfusion, infection, and abnormal placentation such as placenta previa and placenta accreta)..$^{7-9}$ Although there is no universally agreed upon discriminatory point, evidence suggests that women with at least a $60-70 \%$ likelihood of achieving a VBAC who attempt 
TOLAC experience the same or less maternal morbidity than women who have an ERCS. ${ }^{10,11}$ These are the scientific proven reasons why we look forward to give more number of successful TOLAC in our settings and encouraging the same in other tertiary care centers in India.

\section{Materials and Methods}

This is a prospective, cohort study carried out in the labor theatre, of G Kuppuswamy Naidu Memorial Hospital, for a period of 12 months from December 2013 to December 2014. All the patients with previous cesarean section admitted in our hospital for safe confinement were chosen. Among this group, patients who gave consent for the study and satisfied the inclusion criteria were selected as the study sample.

Women with previous one lower transverse cesarean section, singleton fetus in cephalic presentation with clinically adequate pelvis, were included in the study. Women with more than one cesarean section, classical cesarean scar, previous myomectomy, malpresentation, and interdelivery interval less than 2 years were excluded.

VBAC counseling was given after confirming the patients' eligibility for TOLAC. Eligible women were given an information sheet during pregnancy, and written and informed consent was obtained. This study was a patient preference study. Women were allocated based on their preference for either TOLAC or ERCS. We have included a total of 211 women in our study. Detailed history was taken, and patients who preferred ERCS were taken up for elective cesarean section after completion of 39 weeks. In patients who preferred TOLAC, we waited for spontaneous onset of labor. Induction after 39 completed weeks was done as per our hospital protocol in women who did not go into spontaneous labor. In some patients, induction of labor was considered prior to 37 weeks in the presence of other risk factors. Method of induction was chosen according to Bishop score. All women were closely monitored in labor with one-to-one nursing care. Women who chose to abandon trial of labor halfway were taken up for emergency cesarean section in view of maternal request. The percentage of this group of patients who contribute to the failed TOLAC was also studied. Continuous electronic fetal monitoring was done in active labor. Any maternal or fetal abnormalities were identified and promptly acted on. Emergency cesarean section

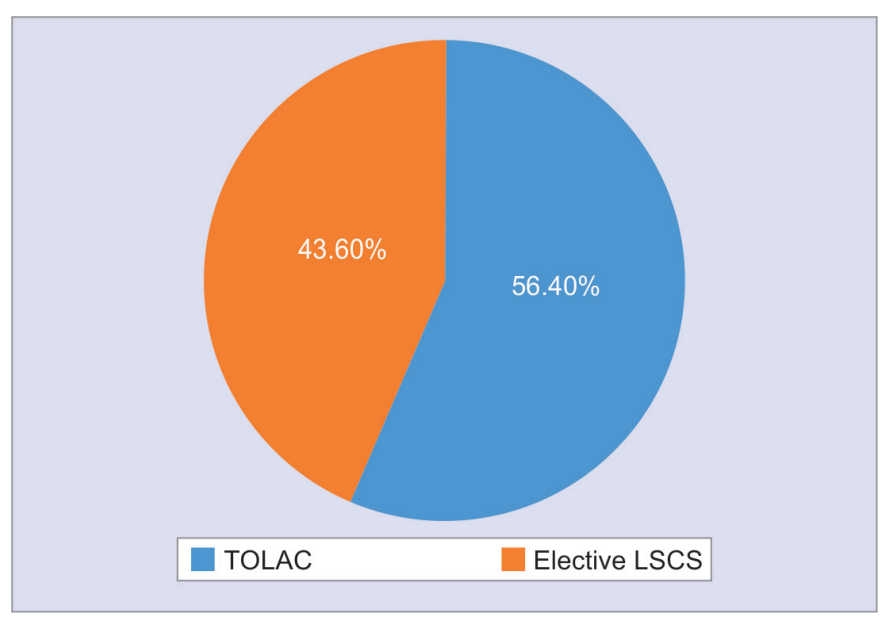

Fig. 1: Mode of delivery was done when necessary. Records were kept about maternal and perinatal complications in labor and after delivery during the period of hospital stay.

The SPSS 18.0 software package was utilized to analyze the data. All values were expressed as mean and standard deviation. We used the Chi-square test to calculate the significance of the association between groups.

\section{Results}

\section{Mode of Delivery}

Out of 211 patients, 119 (56.4\%) were willing for VBAC and had undergone trial of labor, and 92 (43.6\%) were taken up for elective cesarean section in view of maternal request (Fig. 1).

\section{Success Rate of VBAC}

Out of 119 patients, 57 had a successful VBAC (47.9\%) and 62 had an emergency lower segment cesarean section (LSCS) (Fig. 2).

\section{Maternal Complications}

Although maternal complications of uterine rupture, postoperative infection, and scar dehiscence were found to be high in the trial of labor group, $p$ value (0.347) is not found to be significant (Table 1).

\section{Perinatal Complications in the TOLAC Compared to the ERCS Group}

Respiratory distress was found in six neonates (5\%) in the TOLAC group and four neonates (4.3\%) in the ERCS group. There was no case of neonatal hypoxic ischemic encephalopathy (HIE), transient tachypnea of newborn, birth trauma, or any other serious neonatal morbidity and mortality seen in the study group. $p$ value is not found to be significant ( $p=0.814$ ) (Table 2).

\section{Apgar Scores at the End of 5 Minutes in the Trial of Labor Group were Compared to the Elective Cesarean Group}

In the trial of labor group among 119 patients, 6 neonates (5\%) are found to have an Apgar score less than 7 at the end of 5 minutes. In ERCS, five neonates (5.4\%) are found to have an Apgar score less than 7 at the end of 5 minutes. $p$ value is not found to be significant ( $p=0.899$ ) (Table 3).

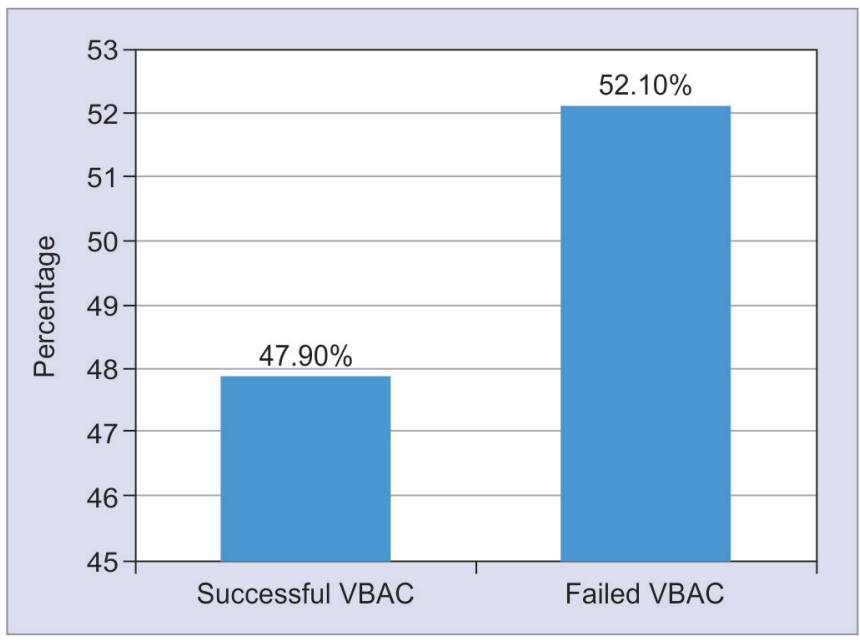

Fig. 2: Success rate of VBAC 


\begin{tabular}{|c|c|c|c|c|c|c|}
\hline & & Nil & $\begin{array}{l}\text { Uterine } \\
\text { rupture }\end{array}$ & $\begin{array}{c}\text { Postoperative } \\
\text { infection }\end{array}$ & Scar dehiscence & Total \\
\hline \multirow[t]{3}{*}{ TOLAC } & Frequency & 113 & 1 & 2 & 3 & 119 \\
\hline & Percentage within TOLAC & 95 & 0.8 & 1.7 & 2.5 & 100 \\
\hline & Percentage within maternal complications & 55.4 & 100 & 66.7 & 100 & 56.4 \\
\hline \multirow[t]{3}{*}{ ERCS } & Frequency & 91 & 0 & 1 & 0 & 92 \\
\hline & Percentage within ERCS & 98.9 & 0 & 1.1 & 0 & 100 \\
\hline & Percentage within maternal complications & 44.6 & 0 & 33.3 & 0 & 43.6 \\
\hline \multirow[t]{3}{*}{ Total } & Frequency & 204 & 1 & 3 & 3 & 211 \\
\hline & Percentage within study group & 96.7 & 0.5 & 1.4 & 1.4 & 100 \\
\hline & Percentage within maternal complications & 100 & 100 & 100 & 100 & 100 \\
\hline
\end{tabular}

Table 2: Perinatal complications in the TOLAC compared to the ERCS group

\begin{tabular}{llccc}
\hline & & \multicolumn{2}{c}{ Perinatal complications } & \\
\cline { 3 - 4 } & & Nil & Respiratory distress & Total \\
\hline \multirow{2}{*}{ TOLAC } & Frequency & 113 & 6 & 119 \\
& Percentage within TOLAC & 95 & 5 & 100 \\
& Percentage within perinatal complications & 56.2 & 60 & 56.4 \\
\multirow{2}{*}{ ERCS } & Frequency & 88 & 4 & 92 \\
& Percentage within ERCS & 95.7 & 4.3 & 100 \\
\multirow{2}{*}{ Total } & Percentage within perinatal complications & 43.8 & 40 & 43.6 \\
& Frequency & 201 & 10 & 211 \\
& Percentage within study group & 95.3 & 4.7 & 100 \\
& Percentage within perinatal complications & 100 & 100 & 100 \\
\hline
\end{tabular}

Table 3: Apgar scores at the end of 5 minutes in the trial of labor group were compared to the elective cesarean group

\begin{tabular}{|c|c|c|c|c|}
\hline & & \multicolumn{2}{|c|}{ Apgar } & \multirow[b]{2}{*}{ Total } \\
\hline & & $<7$ & $>7$ & \\
\hline \multirow[t]{3}{*}{ TOLAC } & Frequency & 6 & 113 & 119 \\
\hline & Percentage within TOLAC & 5 & 95 & 100 \\
\hline & Percentage within Apgar & 54.5 & 56.5 & 56.4 \\
\hline \multirow[t]{3}{*}{ ERCS } & Frequency & 5 & 87 & 92 \\
\hline & Percentage within ERCS & 5.4 & 94.6 & 100 \\
\hline & Percentage within Apgar & 45.5 & 43.5 & 43.6 \\
\hline \multirow[t]{3}{*}{ Total } & Frequency & 11 & 200 & 211 \\
\hline & Percentage within study group & 5.2 & 94.8 & 100 \\
\hline & Percentage within Apgar & 10 & 100 & 100 \\
\hline
\end{tabular}

\section{NICU Admissions in TOLAC Group and in ERCS Group} In the TOLAC group, there was 12 NICU admissions (10.1\%), and in ERCS group, there was 9 NICU admissions (9\%) including preterm care. $p$ value is not found to be significant $(p=0.899)$ (Table 4).

\section{Discussion}

In our study, $43.6 \%$ of the patients underwent TOLAC and the success rate of VBAC was found to be $47.9 \%$. Analyzing the maternal outcomes in the study group, no significant maternal morbidities of uterine rupture, postoperative infection, or scar dehiscence noted $(p=0.347)$. There was no significant respiratory distress, neonatal
HIE, transient tachypnea of newborn, birth trauma, or any other serious neonatal morbidity and mortality seen in the study group $(p=0.814)$. There was also no significant difference in Apgar scores and NICU admissions between both the groups.

In a study by Landon et al. ${ }^{12}$ and Stone et al., ${ }^{13}$ successful rates of VBAC between 56 and $80 \%$ are reported. ACOG $2019^{5}$ reported that generally, $60-80 \%$ of TOLAC result in vaginal delivery. In a meta-analysis done in sub-Saharan Africa involving 14 studies, $^{14}$ the success of vaginal birth after cesarean section was $69 \%$. The success rate of VBAC was $47.9 \%$ in the study group.

In a meta-analysis, TOLAC at term was associated with a maternal mortality of 1.9 per 100,000 versus 9.6 per 100,000 for a 
Table 4: NICU admissions in TOLAC group and in ERCS group

\begin{tabular}{llccc}
\hline & & \multicolumn{3}{c}{ Neonatal care } \\
\cline { 3 - 4 } & & Routine & NICU & Total \\
\hline \multirow{2}{*}{ TOLAC } & Frequency & 107 & 12 & 119 \\
& Percentage within TOLAC & 89.9 & 10.1 & 100 \\
& Percentage within neonatal care & 56.3 & 57.1 & 56.4 \\
ERCS & Frequency & 83 & 9 & 92 \\
& Percentage within ERCS & 90.2 & 9.8 & 100 \\
& Percentage within neonatal care & 43.7 & 42. & 43.6 \\
Total & Frequency & 190 & 21 & 211 \\
& Percentage within study group & 90 & 10 & 100 \\
& Percentage within neonatal care & 100 & 10 & 100 \\
\hline
\end{tabular}

repeat $C D$ (relative risk $[R R], 0.27) .{ }^{15}$ In a study by Landon et al., ${ }^{12}$ the percentage of uterine rupture, uterine scar dehiscence, and uterine infection was $0.7,0.7$, and $2.9 \%$, respectively. To the contrary, however, there were no uterine ruptures, $0.5 \%$ uterine scar dehiscence, and $1.8 \%$ uterine infection in the ERCS group. The risk of uterine rupture was higher among women undergoing TOLAC, but the absolute risk was small only 7 per 1,000. On meta-analysis, the rate of uterine rupture for all women with a history of one previous cesarean section is $0.3 \%$, and the rate of uterine rupture is significantly higher for women who undergo TOLAC compared with ERCS $(0.47$ vs $0.026 \% ; p<0.001) .{ }^{16}$ Most of the high-quality studies on uterine rupture are not stratified by spontaneous versus induced labor, thereby limiting the conclusions that can be made from their results. ${ }^{16}$ In our study group, there was one case $(0.8 \%)$ of uterine rupture, three cases $(2.5 \%)$ of uterine scar dehiscence, and two cases (1.7\%) of postoperative infection. In the ERCS group, there was one case (1.1\%) of postoperative infection with no uterine rupture or scar dehiscence. Although maternal complications of uterine rupture, postoperative infection, and scar dehiscence were found to be high in TOLAC, $p$ value $(0.347)$ is not found to be significant. There was no maternal death or other serious maternal complications in the study group.

The perinatal mortality for TOLAC is 1.3 deaths per 1,000 deliveries, and 0.5 deaths per 1,000 deliveries for women undergoing a repeat CD for an RR of 1.82 (95\% Cl, 1.24-2.76; $p=5.041)$. The incidence of HIE was higher in both low-risk $(0.89 \%$ for TOL vs $0.32 \%$ for repeat CD) and high-risk (1.29\% for TOL vs $0.20 \%$ for repeat $(D)$ pregnancies, but the significance of this difference was not assessed. ${ }^{16}$ In the same study, the need for bag-and-mask ventilation for neonatal respiratory distress was found to be significantly higher following a TOL $(5.4 \%$; $95 \% \mathrm{Cl}, 3.5-7.6 \%)$ than $\operatorname{ERCS}(2.5 \% ; 95 \% \mathrm{Cl}, 0.72-5.0 \%)$. Differences in neonatal intubation rates also could not be assessed. ${ }^{16}$ Landon et al. ${ }^{12}$ found that rates of stillbirth $(0.08 \%)$ and HIE $(0.08 \%)$ were significantly greater in the trial of labor arm. In the present study, respiratory distress was $5 \%$ in the trial of labor group and $4.3 \%$ in ERCS group. There was no case of neonatal HIE, transient tachypnea of newborn, birth trauma, or any other serious neonatal morbidity and mortality seen in the study group. Chi-square value for the association between neonatal morbidity and mortality in TOLAC and in ERCS group is not found to be significant ( $p=0.814)$. Apgar scores, although not always predictive of HIE, can often be used to identify at-risk infants. In a study by Fisler et al. and Kamath et al., ${ }^{15,17}$ Apgar scores were not consistently different in infants delivered by TOLAC versus ERCS. Flamm et al. ${ }^{18}$ found no significant differences in 5 minute Apgar scores or neonatal intensive care unit (NICU) admissions when infants delivered by TOLAC are compared with those delivered by ERCS. In the trial of labor group, 5\% were found to have an Apgar score less than 7 at the end of 5 minutes. In ERCS group, 5 neonates (5.4\%) were found to have an Apgar score less than 7 at the end of 5 minutes. $p$ value is not found to be significant $(p=0.899)$. When assessing the relationship between method of delivery and neonatal admission to the intensive care unit, most trials found no difference between TOLAC and ERCS. ${ }^{16}$ In the trial of labor group, there was $10.1 \%$ NICU admissions, and in elective LSCS group, there was $9 \%$ NICU admissions including preterm care. Based on NICU admissions, Chi-square value (0.005) for the comparison of neonatal risk in TOLAC and in ERCS is not found to be significant ( $p=0.899)$.

\section{Limitations}

It is a short-term study; therefore, long-term complications of ERCS, such as bowel and bladder adhesions, and future pregnancy complications could not be studied.

\section{Conclusion}

The success rate of VBAC was found to be $47.9 \%$ in the study sample. This difference might be due to change in characteristics of obstetric population, acceptance and perseverance of the women to undergo TOLAC, and their mental strength to withstand labor. It also depends upon the hospital resources, expertise, and availability of the obstetrician, as well as the counseling and support to the patient throughout pregnancy and labor. There is no statistically significant risk of maternal and perinatal complications. This might be due to early intervention and delivery. Hence, VBAC is found to be safe with no significant risk of maternal and perinatal complications when done in a tertiary care center following hospital guidelines.

\section{Clinical Significance}

Although VBAC was found to be successful in developed countries, we need more of studies in our Indian setup with the local population to improve the quality of health care and create awareness among patients. This will help us to know the success rate of VBAC, maternal, and neonatal outcome with the available resources in the Indian tertiary care setup. This also helps to compare the characteristics and outcome in the Indian population to that of other countries. In a low-resource setting in India, TOLAC is a nightmare. This present study might boost the obstetricians in the tertiary care setup to counsel more for TOLAC. Instead of opting for ERCS at all levels of health care providers, women willing for VBAC should be counseled and referred for institutional deliveries to reduce the complications of repeat cesarean.

\section{Ethical Approval}

The study was approved by the Institutional Ethics Committee.

\section{References}

1. National Institutes of Health Consensus Development Conference Panel. National Institutes of Health Consensus Development conference statement: vaginal birth after cesarean: new insights March 8-10, 2010. Obstet Gynecol 2010;115(6):1279-1295. DOI: 10.1097/AOG.0b013e3181e459e5.

2. Hamilton B, Martin J, Ventura S. Births: preliminary data for 2009. National Statistics Reports. vol. 59. Hyattsville, MD: National Center for Health Statistics; 2010. 
3. Zhang J, Troendle J, Reddy U, et al. Contemporary cesarean delivery practice in the United States. Am J Obstet Gynecol 2010;203(4):326. e1-326.e10. DOI: 10.1016/j.ajog.2010.06.058.

4. Sargent J, Caughey AB. Vaginal birth after cesarean trends: which way is the pendulum swinging? Obstet Gynecol Clin North Am 2017;44(4):655-666. DOI: 10.1016/j.ogc.2017.08.006.

5. ACOG Practice Bulletin No. 205: Vaginal Birth after Cesarean delivery. Obstet Gynecol 2019;133(2):e110-e127. DOI: 10.1097/ AOG.0000000000003078.

6. Curtin SC, Gregory KD, Korst LM, et al. Maternal morbidity for vaginal and cesarean deliveries, according to previous cesarean history: new data from the birth certificate, 2013. Natl Vital Stat Rep 2015;64(4):1-13 [Level III]. PMID: 26046963.

7. Silver RM, Landon MB, Rouse DJ, et al. Maternal morbidity associated with multiple repeat cesarean deliveries. National Institute of Child Health and Human Development Maternal-Fetal Medicine Units Network. Obstet Gynecol 2006;107(6):1226-1232 [Level II-2]. DOI: 10.1097/01.AOG.0000219750.79480.84.

8. Ananth CV, Smulian JC, Vintzileos AM. The association of placenta previa with history of cesarean delivery and abortion: a meta-analysis. Am J Obstet Gynecol 1997;177(5):1071-1078 [Meta-Analysis]. DOI: 10.1016/s0002-9378(97)70017-6.

9. Nisenblat V, BarakS, Griness OB, etal. Maternal complications associated with multiple cesarean deliveries. Obstet Gynecol 2006;108(1):21-26 [Level II-2]. DOI: 10.1097/01.AOG.0000222380.11069.11.

10. Cahill AG, Stamilio DM, Odibo AO, et al. Is vaginal birth after cesarean (VBAC) or elective repeat cesarean safer in women with a prior vaginal delivery? Am J Obstet Gynecol 2006;195(4):1143-1147 [Level II-2]. DOI: 10.1016/j.ajog.2006.06.045.

11. Grobman WA, Lai Y, Landon MB, et al. Can a prediction model for vaginal birth after cesarean also predict the probability of morbidity related to a trial of labor? Eunice Kennedy Shriver National Institute of Child Health and Human Development Maternal-Fetal Medicine Units Network. Am J Obstet Gynecol 2009;200(1):56.e1-56.e6 [Level II-3]. DOI: 10.1016/j.ajog.2008.06.039.

12. Landon MB, Hauth JC, Leveno KJ, et al. National Institute of Child Health and Human Development Maternal Fetal Medicine Units Network. Maternal and perinatal outcomes associated with trial of labour after prior caesarean delivery. NEJM 2004;351(25):2581-2589. DOI: 10.1056/NEJMoa040405.

13. Stone C, Halliday J, Lumley J, et al. Vaginal birth after caesarean (VBAC): a population study. Paediatr Perinat Epidemiol 2000;14(4):340-348. DOI: 10.1046/j.1365-3016.2000.00299.x.

14. Boulvain M, Fraser WD, Brisson-Carroll G, et al. Trial of labour after caesarean section in sub-Saharan Africa: a meta-analysis. Br J Obstet Gynaecol 1997;104(12):1385-1390. DOI: 10.1111/j.14710528.1997.tb11008.x.

15. Fisler RE, Cohen AC, Ringer SA, et al. Neonatal outcome after trial of labor compared with elective repeat cesarean section. Birth 2003;30(2):83-88. DOI: 10.1046/j.1523-536x.2003.00225.x.

16. Guise J-M, Eden K, Emeis C, et al. Vaginal birth after cesarean: new insights. Evidence report/technology assessment no.191. (Prepared by the Oregon Health and Science University Evidence-based Practice Center under contract no. 290-2007-10057-I). AHRQ Publication No. 10-E003. Rockville (MD): Agency for Healthcare Research and Quality; 2010.

17. Kamath BD, Todd JK, Glazner JE, et al. Neonatal outcomes after elective cesarean delivery. Obstet Gynecol 2009;133(6):1231-1238. DOI: 10.1097/AOG.0b013e3181a66d57.

18. Flamm BL, Geiger AM. Vaginal birth after caesarean delivery: an admission scoring system. Obstet Gynecol 1997;90(6):907. DOI: 10.1016/s0029-7844(97)00531-0. 